\title{
Why Does the Type of Halogen Atom Matter for Radiosensitizing Properties of 5-Substituted 4-Thio-2'-Deoxyuridines? ${ }^{\dagger}$
}

\author{
Paulina Spisz ${ }^{1}$, Magdalena Zdrowowicz ${ }^{1}$, Samanta Makurat ${ }^{1}$, Witold Kozak ${ }^{1}$, \\ Konrad Skotnicki ${ }^{2}$, Krzysztof Bobrowski ${ }^{2}$ and Janusz Rak ${ }^{1, *}$ \\ 1 Laboratory of Biological Sensitizers, Faculty of Chemistry, University of Gdańsk, Wita Stwosza 63, 80-308 \\ Gdańsk, Poland \\ 2 Centre of Radiation Research and Technology, Institute of Nuclear Chemistry and Technology, Dorodna \\ 16, 03-195 Warsaw, Poland \\ * Correspondence: janusz.rak@ug.edu.pl \\ + Presented at the 2nd Molecules Medicinal Chemistry Symposium (MMCS): Facing Novel Challenges in \\ Drug Discovery, Barcelona, Spain, 15-17 May 2019.
}

Published: 8 August 2019

Keywords: radiosensitizers; stationary radiolysis; pulse radiolysis; modified nucleosides; dissociative electron attachment

In order to explain the unexpected difference between radiosensitizing properties of the two studied derivatives, an experimentally-theoretical study combining stationary and pulse radiolysis with quantum-mechanical calculations was carried out. Thus, the extent of radiolysis as well as the identity and amount of stable products were assayed with the HPLC and LC-MS methods. Furthermore, pulse radiolysis experiments enabled the kinetics and spectra characteristics of transients to be characterized. The experimental results were complemented with density functional theory (DFT) calculations on the thermodynamics and kinetics of dissociative electron attachment (DEA) to the studied compounds and possible secondary reactions.

Our results indicate that the difference observed in the cellular experiments can be attributed to different activation barriers of the DEA process. Indeed, the dissociation of the 5-iodo-4-thio-2'deoxyuridine anion is a swift process leading to the reactive 4-thio-5-uridyl radical which, if formed in DNA, may lead to serious damage. On the other hand, a larger activation barrier for DEA to 5-bromo4-thio-2'-deoxyuridine prevents the release of the bromide anion due to competing processes, such as electron autodetachment, protonation, etc.

Funding: This work was supported by the National Science Center (Poland) under the Grant No. UMO2014/14/A/ST4/00405 (J.R.). Calculations were performed in the Wroclaw Center for Networking and Supercomputing, grant No. 209.

(C) 2019 by the authors. Licensee MDPI, Basel, Switzerland. This article is an open access article distributed under the terms and conditions of the Creative Commons Attribution (CC BY) license (http://creativecommons.org/licenses/by/4.0/). 